\title{
Slip localization and fatigue crack nucleation near a non-metallic inclusion in polycrystalline nickel-based superalloy
}

Tiantian Zhang ${ }^{1,}$, Jun Jiang ${ }^{1}$, Barbara A. Shollock ${ }^{1,2}$, T. Ben Britton ${ }^{1}$, Fionn P. E. Dunne ${ }^{1}$

${ }^{1}$ Department of Materials, Imperial College London, London SW7 2AZ, UK

${ }^{2}$ WMG, University of Warwick, Coventry CV4 7AL, UK

*email of corresponding author: Tiantian.zhang08@imperial.ac.uk

This is an authors' version of a publication in Materials Science and Engineering: A Volume 641, 12 August 2015, Pages 328-339 (http://dx.doi.org/10.1016/j.msea.2015.06.070 )

\section{Abstract}

Fatigue crack nucleation at a non-metallic agglomerate inclusion has been studied by high spatial resolution Digital Image Correlation (HR-DIC) and high angular resolution Electron Backscatter Diffraction (HR-EBSD). Spatial and temporal characterization and correlation of deformation with underlying microstructures has been performed, with distributions of plastic strain measured from HR-DIC; and residual stress and density of geometrically necessary dislocations (GND) measured from HR-EBSD. Initial residual stress and GND fields, as a consequence of differing thermal expansivities in the metallic and oxide phases, localized around the agglomerate have been quantified using HR-EBSD. The localization of the pre-existing stress and dislocation states appear to lead to early onset of plasticity upon subsequent mechanical loading. Heterogeneous distributions of plastic strain have been observed in the course of the fatigue test by HR-DIC. Crack nucleation via agglomerate/nickel interface decohesion and particle fracture has been demonstrated and this is correlated with the elevation in strain and dislocation density. The measurements of residual stress, strain, and dislocation density provide key information for the mechanisms of fatigue cracking and the development of damage nucleation criteria in these material systems. 
Key words: fatigue crack nucleation; HR-EBSD; HR-DIC

\section{Introduction}

Understanding fatigue life in metallic components used in gas turbine engines is very important so that effective maintenance schedules can be developed, reducing costs and improving safety. In the engine, polycrystalline nickel based superalloys are widely used for turbine disk applications due to their superior mechanical properties and corrosion resistance at high temperatures. The presence of fine $\gamma$ grains along with a multi modal distribution of $Y^{\prime}$ renders this class of nickel alloys exceptional properties. Evolution of these alloys has involved careful control of chemistry for a wide variety of chemical constituents and this has driven wrought disc components to be manufactured via powder metallurgy routes. This processing route can lead to the introduction of small non-metallic inclusions, despite best efforts to ensure powder cleanliness. These non-metallic inclusions result in degradation in the mechanical properties and understanding their effects on microstructural crack nucleation and short crack growth is very important [1].

Extensive analyses have been carried out to understand the effects of such defects on fatigue crack nucleation and growth in superalloys under different loading conditions and in various microstructures $[2,3]$. However, these experimental observations, albeit comprehensive, do not fully provide a physically sound description of microstructurally sensitive damage initiation and evolution associated with the inclusions. This is complicated further as these inclusions are formed in different manufacturing intervals, and have various chemical compositions, morphologies, and neighboring metallic matrices [2, 4]. This variance challenges the predictive capability of damage nucleation models and therefore the associated damage process needs to be explicitly addressed using more sophisticated techniques. 
The presence of these inclusions, often oxides, in a metallic matrix results in a multiphase material in which damage usually occurs via particle fracture or inclusion/matrix interface decohesion or a mixed mode, depending on the strength of interface, fracture toughness of oxide particle, and plastic response of the matrix [5-7]. This problem is complex and development of physically-based damage nucleation criteria requires microstructurally sensitive characterization of local damage, including plastic and elastic strain distributions, and dislocation content in the vicinity of the inclusion. These quantities evolve during cyclic deformation and lead to crack formation. This necessitates collection and evaluation of high fidelity, high resolution structural data over time.

Local strain evaluation can be performed through direct measurement of plastic strains via Digital Image Correlation (DIC), as well as elastic strains and plastic strain gradients via high angular resolution electron backscatter diffraction (HR-EBSD). These techniques provide complementary, but different, components of the deformation and can be used to inform physical models.

DIC compares two or more images of progressively deformed microstructures to measure surface displacement fields which can be interrogated to measure strains. Typically the displacement resolution and subset size limit this technique to measurements of plastic strain fields [8]. DIC has been used to investigate damage nucleation associated with microstructures and has been conducted in $\mathrm{Ti}$ alloys [9, 10], as well as nickel based superalloy Hastelloy X [11], and recent developments of the technique are reviewed in [8]. Digital image correlation follows surface displacements using images and therefore it is only limited by the imaging quality and patterning size, making it suitable for strain measurement at small scales [12]. In practice, strain resolution is limited by marker size, distribution and contrast within the cross-correlated subsets; and spatial resolution is limited by marker size and imaging mode. Correlation of measured strains with EBSD orientation mapping enables evaluation of the effect of microstructure on strain patterning to be undertaken [11]. However, the total plastic strain field is not the only factor that 
controls crack nucleation and short crack growth and therefore additional techniques are required.

High angular Resolution, cross correlation based, Electron Backscatter Diffraction (HR-EBSD) provides a powerful tool for analyses of residual elastic strain (i.e. stress), lattice misorientation and estimates of the stored geometrically necessary dislocation (GND) density. A recent review provides details of the method [13] and here we describe the method only briefly. This technique measures the relative misorientation and elastic strain state between two or more EBSD patterns. The shifts between zone axes are measured using cross correlation with very high precision and related to the displacement gradient tensor $[14,15]$ with a sensitivity of $\sim 1 \times 10^{-4}$ in strain and $\sim 1 \times 10^{-4}$ rad in rotation [15]. Lattice curvatures from maps of these fields can be calculated and used to generate stored GND content [16].

Recent examples of application of the HR-EBSD technique include the study of GND evolution in Ti-6Al-4V deformed in tension and under cyclic loading [17, 18], slip band-grain boundary interaction in commercially-pure titanium $[19,20]$, and distribution of intragranular residual stresses and GND density in monotonically deformed copper [21-23]. These fields have been used to compare with high fidelity finite element models, including indents in titanium [24]. More recent work on a directionally solidified and a polycrystalline nickel superalloy has shown that the high resolution EBSD determined thermal residual strains and geometrically necessary dislocations are in good agreement with crystal plasticity finite element predications [16, 25-27]. Unfortunately it is only possible to measure the relative deformation state between points within the same grain using HR-EBSD. This has motived a recent study that has involved direct comparison of measurements and crystal plasticity simulations of a thermally induced deformation around an inclusion using the inverse referencing methodology [26]. 
The present work investigates a polycrystalline nickel base superalloy with a non-metallic agglomerate inclusion surrounded by large nickel grains. The cyclic evolution of strains and dislocation densities are analyzed both qualitatively and quantitatively using HR-EBSD and HR-DIC and correlated with key microstructural features. The results are discussed and provide fundamental information for the development of defect nucleation criteria.

\section{Experimental}

\subsection{Material and sample preparation}

A three-point bend cyclic test was conducted on a polycrystalline nickel superalloy (RR1000) sample provided by Rolls-Royce plc. The alloy has a nominal composition as listed in Table 1 [28] and was produced via a powder metallurgy (PM) route. Following extrusion and forging, the alloy was heat treated at a sub-solvus temperature of $1393 \mathrm{~K}$ for four hours and air quenched. This gave rise to the formation of a fine dispersion of intragranular $\gamma^{\prime}$ precipitates at $Y$ phase boundaries. The material was subsequently aged at $1033 \mathrm{~K}$ for sixteen hours. This heat treatment process resulted in a fine grain microstructure and multimodal distribution of $\gamma^{\prime}$ precipitates with increased low cycle fatigue properties. An optical image of the alloy is shown in Fig. 1, together with an orientation EBSD map.

\begin{tabular}{|l|l|l|l|l|l|l|l|l|l|l|l|}
\hline element & $\mathrm{Ni}$ & $\mathrm{Co}$ & $\mathrm{Cr}$ & Mo & $\mathrm{Ta}$ & $\mathrm{Ti}$ & $\mathrm{Al}$ & $\mathrm{B}$ & $\mathrm{C}$ & $\mathrm{Zr}$ & $\mathrm{Hf}$ \\
\hline wt\% & bal. & 18.5 & 15 & 5 & 2 & 3.6 & 3 & 0.015 & 0.027 & 0.06 & 0.5 \\
\hline
\end{tabular}

Table 1 Nominal composition of the nickel base superalloy RR1000 [28]

During the PM processing, contact and reactions between the lining materials and the molten alloying elements are inevitable and are considered to be the source for non-metallic inclusions [2, 4]. In heat treated forgings, these inclusions can be surrounded by large $Y$ grains, as seen in Fig. $1 \mathrm{a}$ and $\mathrm{b}$. 
The as-received specimen was cut down to a rectangular bar with dimensions of $12.70 \times 1.94 \times 3.50 \mathrm{~mm}$ as shown in Fig. 2 (a) based on the loading capacity of the three-point push-push reversible loading rig. Metallographic grinding and polishing were conducted to obtain a surface finish for scanning electron microscopy (SEM). A final light polish with colloidal silica for fifteen minutes was performed to achieve the required surface quality for EBSD. The sample was carefully machined to locate the agglomerate inclusion at the bottom of the test sample which is the preferential site for crack nucleation under the three-point bend configuration depicted in Fig. 2 (b). This region on the front surface is that where the highest macroscopic bending (tensile) stress takes place and hence it is likely to be the location for crack nucleation. The advantage of the three-point bend test over uniaxial or four-point bend testing is that the failure region is localized and identifiable $a$ priori. The region to be investigated, marked in black in Fig. 2 (a), was located using fiducial Vickers micro hardness indents. Prior to the mechanical test, material characterization by means of EBSD was conducted to identify phase boundaries and obtain crystallographic orientations that fall within the investigated region.

\subsection{GND and Residual Stress by High Resolution EBSD}

Significant thermal residual strains and stresses develop at the nickel/agglomerate interface following cooling from aging temperature to room temperature due to differences in coefficients of thermal expansion [26]. The thermal strains are large enough to cause the onset of plastic strain gradients at the interface and hence lattice curvatures. The presence of the curvatures is accommodated by an additional dislocation content, namely that of geometrically necessary dislocations (GNDs). In order to capture the elastic strains and GND densities, cross-correlation based high resolution EBSD was conducted on the area containing the inclusion agglomerate with a size of $60 \times 40 \mu \mathrm{m}$ using a Bruker eflash ${ }^{\text {HR }}$ detector and a Zeiss Auriga SEM. Diffraction patterns at each interrogation point were collected with a probe current of $\sim 13 \mathrm{nA}$ using an exposure time of $200 \mathrm{~ms}$ at full detector resolution (i.e. no binning) $1600 \times 1200$ pixels and a $0.3 \mu \mathrm{m}$ step size between mapped points. 
Cross correlation was performed using in-house scripts with pattern remapping and robust fitting $[29,30] .30$ dispersed regions of interest (ROI) of $256 \times 256$ pixels were used to calculate the full displacement gradient tensor, applying a traction free $\left(\sigma_{z z}=0\right)$ boundary condition and anisotropic Hooke's law with $C_{11}=249 \mathrm{GPa}, \mathrm{C}_{12}=152 \mathrm{GPa}, \mathrm{C}_{44}=124 \mathrm{GPa}$. Afterwards, the fully-resolved 3D tensor was used to obtain elastic strain and lattice rotation components via a polar decomposition and the Green's strain and related stress tensors are reported. Lattice rotations are used to calculate a lower bound estimate of density of GNDs using five of nine components, and one difference, of Nye's tensor [31] for GND density and a $L^{1}$ based line energy minimization scheme [32]. This provides densities of GNDs that support the measured lattice curvatures but this may not be unique.

Suspect data points which had a cross correlation peak height less than 0.3 and a mean angular error greater than $4 \times 10^{-3}$ (first and second passes) were removed.

The GND density recovered by the HR-EBSD technique stems from a reversed Nye's analysis, in which lattice curvature measured between interrogation points are used to construct the Nye's tensor and yield a lower bound GND density. Dislocations with opposite dipoles will 'annihilate' each other, resulting in the absence of lattice curvature and hence will not be measured as geometrically necessary dislocations, but as statistically stored dislocations (SSDs). Therefore, the differentiation between GND and SSD depends on the Burger's circuit size, which is the step size used in EBSD. Opposite dislocation dipoles separated by a sufficiently small Burger’s circuit (step size) will yield higher GND density.

Binning process combines the signal (charge) from a number of adjacent pixels, resulting in an increase in frame rate and signal to noise ratio, while sacrificing the spatial resolution. In current work, no binning was used during the acquisition of diffraction patterns. The noise level associated with the selected binning has been fully justified and considered small [33]. 


\subsection{Plastic Strain Assessment with High Resolution DIC}

After the initial HR-EBSD mapping, a random speckle pattern of fine colloidal silica particles was applied to the region of interest (Fig. 3 a). A reference secondary electron image was captured in this undeformed state with a $100 \times 75 \mu$ m field of view with $3072 \times 2304$ pixels, using $25 \mathrm{kV}$ accelerating voltage and $5 \mathrm{~mm}$ working distance. Brightness was kept at 0 whilst contrast was gradually increased until white silica particles appeared on a black background.

After pre-selected intervals of $2,20,200,1000,1600$, and 1900 cycles, the sample was removed from the loading rig and imaged again for the DIC measurement. The inclusion region was imaged under the same microscope conditions to minimize the effects of change in topographic features on local pixel intensity. To perform the correlation, the images were divided into sub-sets of selected areas, which were individually related to the reference to give in-plane displacements using an in-house image based cross-correlation method (similar to the HR-EBSD cross correlation functions). A band pass filter capable of removing high and low frequency noise raised from background variation was applied.

Components of in-plane strains were obtained by differentiating the displacement field. The size of the colloidal silica particles (50 to $250 \mathrm{~nm}$ ) allowed SEM imaging at high magnification. Compared with the average grain sizes $(\sim 10-15 \mu \mathrm{m}$ for fine grains and $\sim$ tens of $\mu \mathrm{m}$ for the surrounding grains), the applied subset sizes are much smaller, enabling subgrain strain resolution to be achieved.

To assess the noise level associated with each set of the employed DIC parameters, two consecutive images with a small amount of rigid body translation were captured at the initial step. Image correlation of this pair of images provides an approximation of the error in the strain measurement, raised from the drift effect during raster scanning and detector sensitivity. The systematic error decreases for increasing subset size with a compromise in the resolution. Since the first two cycles introduced larger plastic strains in the scanned area (see later), different DIC subset sizes were used (Fig. 3b). In this sense, subset sizes of 
$\sim 4.9 \times 4.9 \mu \mathrm{m}^{2}$ and $\sim 1.72 \times 1.72 \mu \mathrm{m}^{2}$ were employed for the $0-2$ cycle interval, and the remaining intervals, respectively. This corresponds to a spatial resolution of $0.032 \mu \mathrm{m} / \mathrm{pixel}$. The corresponding root mean square errors are 0.0021 (0-2 cycle interval) and 0.0043 (remaining intervals) which are magnitudes lower than those calculated strains (shown later) and therefore no correction has been implemented. The reported errors are associated with the $x x$ direction. Since strains in other directions are small and not explicitly displayed, the corresponding errors are omitted. An overlap of $90 \%$ was used throughout the DIC analysis. As a result, the chosen DIC parameters yield acceptable noise level and strain resolution.

\subsection{Three-point bend testing}

After the initial characterization, application of the speckle pattern, and acquisition of the reference image, the sample was fatigued under force-controlled loading at a frequency of $0.023 \mathrm{~Hz}$ with a triangular waveform. The test was performed with a maximum force of 2200 $\mathrm{N}$ and a rate of $100 \mathrm{~N} \mathrm{~s}^{-1}$ on a Zwick Roell Z010 load frame. The test was interrupted at selected intervals, and the sample was removed from the rig to capture deformed images for DIC strain measurement, and was examined for evidence of micro cracks, and then reloaded. In Fig. 4 a, the first cycle introduced a significant amount of plasticity as illustrated by the extended plastic region in the load-displacement curve after the initial yielding point. In Fig. 4 b, macro ratcheting occurred at the macroscopic level as indicated by the curve that progressively shifts to greater displacements during subsequent loading.

\subsection{Calculation of accumulated plastic strain}

In a manner similar to other surface strain measurement techniques, DIC only provides strain components, $\varepsilon_{x x}, \varepsilon_{y y}$, and $\varepsilon_{x y}$ for the measured free surface. However, by assuming plastic incompressibility, it is possible to obtain the strain in the out-of-plane direction, $\varepsilon_{z z}$, using the following equation

$\varepsilon_{x x}+\varepsilon_{y y}+\varepsilon_{z z}=0$ 
The shear strains in the third direction remain unknown, but they can be obtained by using the normality hypothesis of plasticity. The stress condition corresponding to the free surface satisfies the following equation

$\sigma_{z z}=\sigma_{x z}=\sigma_{y z}=0$

For a material that satisfies von Mises yield criterion, the plastic strain increment, $\dot{\boldsymbol{\varepsilon}}^{p}$, may be related to the deviatoric stress as

$\dot{\varepsilon}_{i z}^{p}=\frac{3}{2} \frac{p}{\sigma_{e}} \sigma_{i z}^{\prime}=\frac{3}{2} \frac{p}{\sigma_{e}} \sigma_{i z} \equiv 0$

where $\mathrm{i}=\mathrm{x}, \mathrm{y}$, and $\mathrm{z}$, and ${\sigma^{\prime}}_{i z}$ are the out-of-plane components in the deviatoric stress tensor

$\sigma^{\prime}$ given by $\sigma^{\prime}=\sigma-\frac{1}{3} \operatorname{Tr}(\sigma) I$. By assuming the contribution from elastic shear strains to be small, it may be deduced that

$$
\varepsilon_{i z}^{p}=\dot{\varepsilon}_{i z}^{p}=0
$$

As a result, the formulation of the effective plastic strain, $\varepsilon_{e f f}^{p}$, can be simplified into the form of equation 5 .

$\varepsilon_{\text {eff }}^{\mathrm{p}}=\sqrt{\left(\frac{2}{3} \boldsymbol{\varepsilon}^{\mathrm{p}}: \boldsymbol{\varepsilon}^{\mathrm{p}}\right)}=\left[\frac{2}{3}\left(\varepsilon_{x x}^{p^{2}}+\varepsilon_{y y}^{p^{2}}+2 \varepsilon_{x y}^{p^{2}}\right)\right]^{1 / 2}$

\section{Results}

\subsection{Residual stress and GND density by HR-EBSD}

Fig. 5 (a) shows the field plots of the $x x$ stress measured by HR-EBSD after the heat treatment. Some heterogeneities in the surrounding nickel grains are observed, which is largely due to the complex distribution of the agglomerate particles as well as the different crystallographic orientations of the surrounding grains. In HR-EBSD, the elastic strains and stresses are measured with respect to grain unique reference points which are unlikely to be unstrained. Consequently, the displayed stresses in Fig. 5 (a) are relative to those values at grain-unique reference points and make grain-by-grain comparison problematic. Fig. 5 (b) shows stresses after all the cyclic deformation is complete. Type III (intragranular) 
stresses in two selected surrounding grains measured prior to and after the cyclic loading are shown in the insets of Fig. 5 (a) and (b), respectively. In Grain 1, the initial stress distribution is relatively uniform, compared to that in the deformed state where significant concentration at grain boundaries is observed. In Grain 2, the average stress decreases from about $500 \mathrm{MPa}$ (rendered yellow in the field plot) to $\sim 1200 \mathrm{MPa}$ (dark blue).

Fig. $5 \mathrm{c}$ and $\mathrm{d}$ show GND distributions prior to and after the cyclic deformation with insets focusing on the agglomerate region. Prior to the cyclic deformation, it can be observed that the GND distribution is significantly heterogeneous; a large surrounding grain with greater dislocation density is circled in white. Areas of high GND density, rendered red, have densities of order $10^{15} \mathrm{~m}^{-2}$ and above.

The fatigue loading has introduced a significant amount of deformation to the material, resulting in crystallographic slip and lattice rotation. After the cyclic deformation, it can be seen that the overall GND density increases significantly (Fig. $5 \mathrm{~d}$ ). Ni grains within a radius of $\sim 50 \mu \mathrm{m}$ from the agglomerate exhibit GND densities as high as $10^{15} \mathrm{~m}^{-2}$ or above. The GND densities in two selected grains, as illustrated in the insets, show development of significant substructure, particularly in grain 1 where GND accumulation is observed at the top and bottom twin boundaries. It is also interesting to notice that the large surrounding nickel grains and fine grains appear to display equal magnitudes of GND densities. Only in those fine grains that are further away from the agglomerate, can lower GND densities be observed.

Appearing in white, significant numbers of interrogation points have failed to crosscorrelate with the references and give rise to the change in the appearance of the grains. This is associated with the data quality metrics employed by the HR-EBSD measurement and a more detailed discussion is given later.

To further quantitatively analyze the evolution of the stresses that are shown in Fig. 5, the stress histograms for grain 1 and 2 are plotted in Fig. 6 a and b, respectively. Since stress 
measured in HR-EBSD is relative to a reference value, the stress magnitudes presented in Fig. $6 \mathrm{a}$ and $\mathrm{b}$ are obtained by removing grain-average stress from stress at each point. The initial states for both grain 1 and 2 have narrower stress magnitudes compared to that after 1900 cycles. The change in standard deviation of the two selected grains (Fig. $5 \mathrm{c}$ ), representing the range of stress values present increases significantly by a factor of 4 in grain 1, whereas that of grain 2 shows a less profound elevation. Referring back to Fig. $5 \mathrm{~b}$, the maximum values of $x x$ stress in grain 1 appear to segregate to grain boundaries, while grain 2 is deformed more uniformly. The aforementioned quantification reflects appropriately the qualitative assessment shown in Fig. 5.

Fig. 7 (a) shows a histogram of GND densities for the whole map measured before and after the cyclic deformation. At the initial stage (blue bars), the GND density, peaking between $10^{13}$ and $10^{14} \mathrm{~m}^{-2}$, follows approximately a log-normal distribution except for a prolonged tail expanding to about $10^{15} \mathrm{~m}^{-2}$. This can be attributed to the localised plasticity or dislocation accumulation at the agglomerate/nickel interface due to the incompatible deformation as a result of the differing thermal expansivities. The cyclic deformation has elevated the overall dislocation density and resulted in a split of the initial peak. A primary peak has GND density of about $10^{15} \mathrm{~m}^{-2}$, corresponding to those heavily deformed grains near the agglomerate. In contrast, a small secondary peak observed in the histogram reflects those less deformed grains that are away from the agglomerate with GND densities remaining at the same magnitude as those at the undeformed state (between $10^{13}$ and $10^{14}$ $\left.m^{-2}\right)$.

Evolution of GND densities in the two selected grains surrounding the agglomerate is further quantitatively analyzed by plotting their means and standard deviations in Fig. $7 \mathrm{~b}$. The average densities of GNDs increase significantly, particularly in grain 2 where there is an order of magnitude difference. On the other hand, the standard deviation remains almost stable before and after the deformation. Since the maximum GND densities appear to gradually saturate, it may indicate that the GND density at the final deformed state or even 
before has reached a statistical limit of about $10^{15} \mathrm{~m}^{-2}$. However, due to the absence of GND density measurement between fatigue cycles, further evidence is required to fully rationalize the potential saturation of GND density.

\subsection{Development of cyclic total strain patterns by HR-DIC}

Fig. 8 shows the field plots of incremental strains in the $x x$ direction as a function of increasing number of fatigue cycles with insets showing details around the agglomerate marked by the white dashed lines. The scale bars are set to be the same except that for the first two cycles, which has been increased by a factor of two to highlight the strain development. Grain boundaries obtained using EBSD from the initial deformation state are overlaid on top of the strain maps to correlate key microstructural features with the strain localization. These strain maps are obtained by comparing two consecutive DIC images. That is, the strain magnitudes shown for a given cycle correspond to the differences developed since the previous measurement. Hence, the strains shown for cycle 1900 are the strain changes from those measured at cycle 2. A significant level of heterogeneity can be observed in the course of the fatigue loading. After two cycles of fatigue loading, no micro crack is observed in the scanned region. In Fig. 8 (a), some regions with high magnitudes of strain (dark red) can be seen, while some regions appearing green exhibit nearly zero straining. This variation can be related to the susceptibility of the microstructures to local loading conditions, and to local crystallographic orientation. In the region marked by a dashed blue square in Fig 8 a', a curved region with strain concentration of nearly 0.2 is seen. With a further 18 cycles of loading, a micro crack forms at this location via agglomerate/nickel interface decohesion observed by means of SEM. The corresponding strains shown in Fig. 8 (b) and (b'), and all subsequent strain maps are therefore influenced by the formation of the crack since the imposed strains are small compared to those generated by the opening of the crack, and thus no strain concentration is seen in the surrounding grains. A figure showing progressive strain localization after 2, 20, 200, 1000, 1600,1900 cycles is provided in Supplementary. 
The cyclic strains were measured at the unloaded state and are therefore dominated by residual plastic strains. Elastic strains are present as a result of kinematically imposed constraints between neighboring grains, but their magnitudes are small and hence ignored. The contour plot of the effective plastic strain in Fig. 9 (a) shows that the distribution of accumulated plastic strain obtained after two cycles mirrors that of the $x x$ strain in Fig. 8 a, indicating that the axial $x x$ strain dominates.

To elucidate the effect of local microstructure such as the large surrounding grains, fine grains, and agglomerate, on the development of deformation pattern, strains from these microstructural features (Fig. 10 a) are plotted as a function of fatigue cycles in Fig. 10 (bc) at the locations 1 to 5 shown. The strains in Fig. $10 \mathrm{~b}$ are calculated based on correlations between the undeformed state and each deformed state and hence show total strain accumulation at the cycles shown. In contrast, those in Fig. $10 \mathrm{c}$ are incremental and calculated based on correlations between two consecutive images, thus referring to strains developed during $0-2,2-20,20-200,200-1000,1000-1600$, and 1600-1900 intervals, respectively. In Fig. 10 (b), after the first two cycles, a localization close to the agglomerate (point 4) has considerably greater strain than those at the other interrogation points. Point 5 (fine grain) has a strain of about 3.3\%, a factor of two lower compared to the magnitudes calculated for the large surrounding grains (Point 1-3). Nevertheless, strains at all tracked points progressively increase with increasing number of loading cycles, indicating the establishment of local ratcheting. A strain concentration at the agglomerate is clearly observed after the first two cycles. Once a micro crack nucleated between 2-20 cycles, the strain increases sharply and a large contribution to the increase is made by the coalescence of cracks and the subsequent propagation.

To provide a quantitative assessment of strain accumulation at the agglomerate/nickel interface, a line scan running from the agglomerate vicinity towards grain interior is implemented as shown in Fig. 11 (a), along which xx strains along the path are extracted and plotted in Fig. 11 (b). The displayed strains are calculated based on correlations 
between the undeformed state and each deformed state and hence show total accumulated strain. After two cycles of fatigue loading, considerable accumulation is observed not only at the agglomerate/nickel interface, but also near a $\Sigma 3$ twin boundary as indicated by the dashed line in Fig. 11 (a). The former appears to be associated with load transfer from the metallic matrix to the particles, while the latter can be attributed to whether or not the orientation is favorable for slip and grain boundary characteristics which prevent slip transmission. The strain accumulates at a much faster rate in the agglomerate vicinity than that in the grain interior and in the twin boundary. This deformation pattern is established after the first two cycles and remains stable throughout the remaining cycles apart from a strain localization developed readily in the course of the test at a distance of $2 \mu \mathrm{m}$ from the agglomerate. This corresponds to the strains indicated during coalescence of the micro cracks.

After the first two cycles, the agglomerate/nickel boundaries remained undamaged and no cracking was detected at these locations using the electron microscope. With an additional 18 cycles, a micro crack was formed via interfacial decohesion. Further cyclic straining resulted in the formation of several micro cracks by both interfacial decohesion and fracture of oxide particles, which can be seen in Fig. 12. Subsequently, after 1400 cycles, these cracks began to coalesce and propagate into surrounding nickel grains along a path that was macroscopically perpendicular to the $x$ direction in Fig. 2. Throughout the analysis, no fatigue crack nucleation was observed in any nickel grains.

\section{Discussion}

The current work has investigated the evolution of plastic strain, residual stress, and GND density around a complex non-metallic inclusion in a polycrystalline $\mathrm{Ni}$ superalloy under cyclic loading by means of high resolution DIC and EBSD. The heterogeneously-distributed strain, stress, and dislocation density have been correlated with the underlying microstructures to understand the localization of strain and dislocation density and 
subsequently relate to the nucleation of fatigue cracks. The results are of importance for the development of damage nucleation models whose predictive potential depend on accurate capture of the spatial and temporal evolution of local strain, residual stress, and dislocation density at the origin of damage [34-37].

The HR-EBSD technique employed in current study performs image cross-correlation of electron backscattered diffraction patterns in Fourier space, which allows high angular resolution (uncertainty of pattern shift of $\sim 0.05$ pixels in [15]) to be achieved. This high sensitivity differentiates the HR-EBSD from conventional EBSD analysis performed in Hough space, which only allows lower angular resolution (a few pixels for uncertainty in pattern shift). The HR-EBSD itself is an off-line analysis process of the Electron Backscattered Diffraction Patterns obtained using conventional EBSD. Therefore, its spatial resolution depends on the size of the diffraction volume where the backscattered electrons originate. Usually, this size is of an order of tens of nanometers [38].

The residual deformation as a consequence of the thermal loading history can have considerable effects on the damage evolution and failure mechanisms when subjected to the subsequent mechanical loading. The thermal loading from the annealing temperature to room temperature caused the onset of plasticity local to the agglomerate due to differing thermal expansivities. The sample was annealed at $1033 \mathrm{~K}$ for 16 hours to reduce the residual stresses that were introduced during previous mechanical processing. Since the thermal expansivity of nickel is assumed to be isotropic, it is reasonable to argue that the annealed sample is macroscopically stress-free [25]. However, at the agglomerate/nickel boundaries, cooling from the annealing temperature to room temperature results in the development of significant residual stress, lattice rotation, and most likely plasticity by virtue of the differing thermal expansivities.

The initial GND densities near the agglomerate quantified by HR-EBSD are broadly consistent with other observations in similar materials. Karamched and Wilkinson [16] reported an 
average GND density of $\sim 3 \times 10^{14} \mathrm{~m}^{-2}$ at the carbide/nickel interface in a directionally solidified nickel alloy. Zhang et al [26] performed HR-EBSD analysis on non-metallic inclusions in a polycrystalline nickel and revealed a GND density of $\sim 10^{14} \mathrm{~m}^{-2}$. In the Al matrix SiC reinforced metal matrix composite, Arsenault and Shi [39] used TEM to determine a GND density of $\sim 10^{14} \mathrm{~m}^{-2}$ at the SiC/Al interface. The average GND density near the inclusion determined in current study is $\sim 10^{14} \mathrm{~m}^{-2}$, though a dislocation band (circled in white in Fig. $5 \mathrm{c}$ ) along a twin boundary with a magnitude of $\sim 10^{15}$ are observed. It is likely that there is a correlation with the twin boundary, agglomerate, and perhaps the subsurface microstructure.

Cooling from the aging temperature to room temperature leads to the development of strain localization (i. e. residual stress and plastic deformation) due to the difference in the coefficients of thermal expansion. It is expected to observe localized GND densities near the inclusion as a result of the strain gradient. Therefore, the observed differences in detailed distributions of residual stress and GND density between grain 1 and 2 are largely due to the complex shape of the inclusion agglomerate and the distance away from it. Irregular geometries, such as sharp corners and small inter-particle spacing can significantly elevate the stress and strain magnitudes. Crystallographic orientations of the surrounding grains and grain boundary also play a role in the localization.

The corresponding plastic strains and strain gradients resulted in the development of distributions of GND densities which can cause local hardening, as well as local stress elevations from the associated residual stress fields. The elevation of stress field potentially contributes to the interface decohesion and fracture of oxide particles. A grain with greater GND density is observed on the left of the agglomerate in Fig. $5 \mathrm{c}$ (circled in white) and a micro crack formed by interfacial decohesion was first observed at this location. In this context, the quantification of residual stress provided by the HR EBSD potentially aids the understanding of the crack nucleation in the agglomerate region. 
Under the low cycle fatigue condition, the investigated region experienced significant plastic deformation. The deformation mechanism for the nickel grains is crystallographic slip, which can be revealed by the presence of slip lines. A BSE image of the agglomerate region (Figure $9 \mathrm{~b}$ ) shows the slip activity developed. In addition, regions of high residual stress and GND density at grain boundaries can be observed in grain 1 in the EBSD strain and GND density maps (Fig. 5 b and d). Other grains show no apparent features associated with slip due to increased number of unindexed points as a result of the plastic deformation.

Some regions with strain concentration in Figure 9 (a) exhibit no obvious correlation with microstructures. The highly complex microstructures in the investigated region and those underneath the free surface can have significant effects. The contribution from the fine grained region to the strain distribution in the large grains has not been experimentally and numerically studied. In Fig. 9 (b), examination of the surface of the post-deformation sample reveals the development of slip lines in the surrounding grains. These slip activities are caused by the movement of individual dislocations and accumulation at the free surface. The pattern of effective plastic strain in Figure 9 (a) may indicate some evidence of such slip activity. However, strain localization does not necessarily occur in regions near grain boundaries or triple junctions. HR-EBSD analysis in polycrystalline copper has demonstrated that patterns of GNDs are orientation dependent, regardless of proximity to grain boundaries [40]. Grains with different orientations exhibit distinct dislocation structures and therefore this can have an influence on strain distribution. Additional studies, such as 3D analysis to reveal the grain structures below the free surface, would benefit understanding the physical mechanisms.

The cyclic deformation introduced a significant amount of plasticity (Figures $10 \mathrm{~b}$ and c) to the investigated region, resulting in the development of high level of heterogeneity of strain, and hence significant strain gradients and GND densities. Literature considering the development of GND density in uniaxially deformed $\mathrm{Cu}$ [22], $\mathrm{Ti}$ [17], and cyclically deformed Ti [18] up to similar strain amplitudes has reported high GND densities of order of $10^{15} \mathrm{~m}^{-2}$ 
or above at not only grain or/and twin boundaries, and triple junctions, but also grain interior. Grain and twin boundaries can inhibit dislocation motion. However, given the high remote strain applied, it is reasonable to observe a relatively homogeneous distribution of GNDs at a distance of a few grains from the agglomerate. A parallel study considering the effect of inclusion on the localization of dislocations has suggests that GND density increases rapidly near the inclusion to an order of $10^{15} \mathrm{~m}^{-2}$. Further cyclic loading leads to an expansion of this high GND density region in microstructures to a few grains away from the inclusion.

The strain pattern appears to be established at the beginning of the fatigue test, i.e. during the first two cycles and stabilizes until the crack has propagated into the surrounding nickel grains. Throughout Fig. 8 (c-f) and (c'-f'), the strain distribution evolves to include an apparent localization (indicated by white arrow) at the agglomerate region, which progressively elongates in the direction perpendicular to the $\mathrm{x}$ direction and eventually propagates into the surrounding nickel grains. Microscopic examination of the agglomerate reveals that multiple cracks nucleated either by interface decohesion or particle fracture in the course of the fatigue test. As a result, it is argued that the apparent strain localization and propagation is generated by the coalescence and growth of the micro cracks.

Due to the nature of the force-controlled loading, the specimen experienced significant plastic straining during the first cycle and hardened rapidly, resulting in the development of smaller plastic strains in the subsequent cycles. The plastic strain ranges established at the very bottom of the specimen in the subsequent cycles have an average value of $0.25 \%$ or smaller. The remote strain amplitude is obtained by performing a macro DIC measurement on a large area comparable to the size of the beam. Nonetheless, at the microscopic level, local ratcheting with strain magnitudes above $10 \%$ can be observed in the nickel matrix as shown in Fig. 10 and 11. This observation provides direct evidence of the highly localised and heterogeneous strain fields at individual grain level even though the macroscopic applied strain is small. Microstructural features that control strain localisation, 
such as crystallographic orientations of grains and grain boundary characteristics, play an important role in this accumulation.

The distribution of the GNDs is heterogeneous at individual grain level and also at intergranular level as shown in Fig. $5 \mathrm{~d}$. The formation of the GND substructures, i.e. the two dislocation bands in Grain 1, can be attributed to heterogeneous response and plastic strain gradients developed by the local applied stress state. Dislocations accumulate at or transmit through grain boundaries, depending on the grain boundary characteristics and residual Burger's vector [11]. The agglomerate/nickel boundaries, however, inhibit dislocation transmission as the oxide phase experiences limited or no plastic slip. As a result, it is reasonable to expect dislocation pileup at such boundaries. However, it is in these regions where unknown GND densities (appearing white in GND map) are observed. This is related to the removal of unreliable cross correlations of the EBSD patterns near the agglomerate/nickel boundaries. Due to the significant deformation generated, blurring of the EBSD patterns obtained from these regions prevents the robust iterative fitting scheme from obtaining reliable solutions [30]. However, the establishment of lattice rotations near the agglomerate (not shown explicitly) are anticipated to give rise to lattice curvatures and hence accumulation of GNDs towards the phase boundaries.

In Figs. 8-11, some evidence has been provided that the strain accumulation at the agglomerate/nickel interfaces results in the crack formation by interfacial decohesion, which was initially observed after 20 cycles. Once the micro crack had formed, an apparent strain localization in the cracked region was observed, which facilitated further decohesion and cracking activities occurring in its vicinity. The considerable amount of strain localization shown in Fig. $8 b^{\prime} \sim f^{\prime}$ is therefore attributed to the coalescence and propagation of these micro cracks. No damage nucleation has been observed in the nickel polycrystal during the test, indicating the fact that the agglomerate is more susceptible to fatigue crack nucleation. Both particle fracture and interface decohesion mechanisms have been observed 
in the test, indicating a competition which needs to be clarified to determine the conditions under which each mechanism dominates.

The mechanistic process of crack nucleation at inclusion/matrix interface is often characterized by increasing interfacial separation; the traction, defined as force per unit area, across the interface increases, maximizes, and decreases eventually. After reaching a threshold value of separation, complete decohesion occurs. Constitutive equations for cohesive interfaces must take into account the deformation and failure mechanisms, which vary structurally depending on the phases present. The strength of the interface and the work of separation are decisive parameters for the decohesion process. Examples of constitutive equations for inclusion/matrix interface decohesion can be found in numerical parametric studies [41-43], in which cohesive interactions are formulated in an idealized cohesive zone finite element framework with periodic boundary conditions. In these studies, phenomenological interface constitutive equations are employed where the cohesive interactions are assumed to account for atomistically sharp planes only, whereas in reality cohesive interactions can be more complex. Defect nucleation criteria based on both critical stress and/or strain are reported in the literature, such as [44]. However, the critical values are related with respect to overall stress and/or strain states, necessitating local quantities at key interfaces to be assumed, within the heterogeneous distributions of such quantities in polycrystalline materials. The present work provides direct measurements of residual stress and strain at the length scale controlling the damage nucleation process, and thus facilitating development of damage nucleation criteria.

The plastic response of the matrix can have important influence on damage initiation mechanisms. An X-ray tomography study on metal matrix composites has reported that plastic properties of the metallic matrix surrounding an inclusion phase play an important role [5]. Particle fracture prevailed in precipitation-hardened Al matrix with high yield stress and work hardening rate, whereas the mechanism changed to interface decohesion in pure Al. An in-situ synchrotron tomography analysis on damage development in $2080 \mathrm{Al}$ 
strengthened by $\mathrm{SiC}$ particles has revealed that particle fracture was the controlling damage mode [7].

Returning to the present study, Fig. 13 shows the xx plastic strain map at the unloaded state after two cycles with EBSD-determined grain boundaries overlaid and the Schmid factor map assuming a macroscopic tensile $x x$ stress for the initial state. In general, grains with larger Schmid factors (appearing brighter in Fig. 13 b) exhibit higher strain levels, whereas areas with smaller Schmid factors tend to have lower strains. Lower strains at grain or twin boundaries compared to those at the interior are also observed, indicating that grain-tograin compatibility and slip transmission across grain boundaries play a role in the development of strain localization.

However, there are variances of the correlation, which manifest the incapability of predicating local deformation states using this crude global Schmid factor. Due to complex local stress fields, indicated in part by the residual stress fields measured with HR-EBSD, Schmid factor can vary significantly even within a single grain, making some areas which were badly orientated for slip become favorably orientated, and vice versa.

After 20 cycles of straining, a micro crack was observed in the inclusion region and propagated upon further loading. The displacement fields associated with this crack nucleation and propagation give rise to strain accumulation, which can be clearly observed throughout the test as shown in Fig. 8 b-f. However, the displacement fields in the vicinity of the cracks are generated by rigid body displacement as a result of crack opening. This affects the DIC strain measurements near the cracks as shown in Fig. 8, 10-11 and makes them difficult to interpret. A potential way to overcome this problem requires identification of the crack geometry and its size. However, the current DIC technique is not capable of masking the cracked region accurately and hence care must be taken when interpreting the strain measurements around the cracks. 
The studied material is a polycrystalline nickel superalloy heat treated at a subsolvus temperature, resulting in a tri-modal distribution of gamma prime precipitate. No reliable data on the sizes of gamma prime precipitates are available in open literature. Instead, the size distributions in a comparable nickel superalloy (U720Li) are given as follows [45]. The primary gamma prime at the gamma grain boundaries has a mean grain size of 1-10 $\mu \mathrm{m}$. The secondary gamma prime in gamma grains has a size distribution from 70-120 nm. Smaller tertiary gamma prime precipitates can be found in between the secondary gamma prime with tens of nanometers in size. The particle size of the speckle pattern $(\sim 250 \mathrm{~nm})$ is not sufficiently small to capture slip activities that occurred within these phases. However, at room temperature, the effect of the gamma prime precipitates on slip seems to be small and there not considered in current study.

\section{Conclusions}

Fatigue damage nucleation and evolution in a powder metallurgy nickel base superalloy containing a non-metallic agglomerate inclusion has been captured by high resolution electron back scatter diffraction and high resolution digital image correlation. Micro crack formation via decohesion of agglomerate/nickel interface and fracture of oxide particles has been characterized using secondary electron microscopy. The thermal residual deformation from the manufacturing of the alloy led to early onset of plasticity local to the inclusion agglomerate. The subsequent fatigue loading resulted in the establishment of significant plastic strain concentration at the agglomerate as measured by the HR DIC. Though the macroscopic strain was small, heterogeneously developed strain fields were observed with local strain magnitude of above $10 \%$. The HR-EBSD observation indicated a dramatic elevation of GND density and residual stress in the nickel grains surrounding the inclusion. The mechanistic basis for fatigue crack nucleation involves slip accumulation and localization, the establishment of local high GND densities and consequent local stress, giving rise to both oxide particle cracking and particle/matrix decohesion. The damage 
nucleation mechanisms, oxide/nickel interface decohesion and fracture of oxide particle, are likely to be controlled by a combination of critical strain and stress states, potentially coupled in terms of a local stored energy rate measure [36, 37]. The interface strength also remains a key parameter. Quantitative assessment of defect nucleation utilizing crystal plasticity modelling techniques is currently underway.

\section{Acknowledgements}

Tiantian Zhang is grateful for the financial support and supply of nickel superalloy from Rolls-Royce plc. The authors much appreciate helpful discussions Dr Mark Hardy and lain Parr (Rolls-Royce plc.). Useful advice from Zebang Zheng is acknowledged. Ben Britton acknowledges funding from a Small Equipment Grant from EPSRC (EP/K030760/1).

\section{Supplementary}

Fig. 14 shows the field plots of incremental strains in the $x x$ direction as a function of increasing number of fatigue cycles with insets showing details around the agglomerate marked by the white dashed lines. The propagation of the micro crack can be indicated by the progressive increase and elongation of the highly strained region. A more detailed investigation of how microstructures affect the plastic zone ahead of the crack tip and the path of crack propagation would be valuable, but it is not explicitly addressed in current study.

\section{References}

[1] P. Kantzos, P. Bonacuse, J. Telesman, T. Gabb, R. Barrie, A. Banik. Superalloys 2004 (Tenth International Symposium), 2004. p.409-417.

[2] E.S. Huron, P.G. Roth. Superalloys 1996 (1996) 359-368.

[3] F. Alexandre, S. Deyber, A. Pineau. Scr. Mater. 50 (2004) 25-30. 
[4] D.A. Jablonski. Materials Science and Engineering 48 (1981) 189-198.

[5] L. Babout, E. Maire, R. Fougères. Acta Mater. 52 (2004) 2475-2487.

[6] L. Babout, E. Maire, J.Y. Buffière, R. Fougères. Acta Mater. 49 (2001) 2055-2063.

[7] J.J. Williams, N.C. Chapman, V. Jakkali, V.A. Tanna, N. Chawla, X. Xiao, F. De Carlo. Metall and Mat Trans A 42 (2011) 2999-3005.

[8] B. Pan, K.M. Qian, H.M. Xie, A. Asundi. Measurement Science \& Technology 20 (2009).

[9] C. Efstathiou, H. Sehitoglu, J. Lambros. Int. J. Plast. 26 (2010) 93-106.

[10] P.D. Littlewood, A.J. Wilkinson. International Journal of Fatigue 43 (2012) 111-119.

[11] W.Z. Abuzaid, M.D. Sangid, J.D. Carroll, H. Sehitoglu, J. Lambros. J. Mech. Phys. Solids 60 (2012) 1201-1220.

[12] F. Di Gioacchino, J. Quinta da Fonseca. Exp. Mech. 53 (2013) 743-754.

[13] T. Ben Britton, J. Jiang, P.S. Karamched, A.J. Wilkinson. Jom-Us 65 (2013) 12451253.

[14] A.J. Wilkinson, G. Meaden, D.J. Dingley. Materials Science and Technology 22 (2006) 1271-1278.

[15] A.J. Wilkinson, G. Meaden, D.J. Dingley. Ultramicroscopy 106 (2006) 307-313.

[16] P.S. Karamched, A.J. Wilkinson. Acta Mater. 59 (2011) 263-272.

[17] P.D. Littlewood, T.B. Britton, A.J. Wilkinson. Acta Mater. 59 (2011) 6489-6500.

[18] P.D. Littlewood, A.J. Wilkinson. Acta Mater. 60 (2012) 5516-5525.

[19] Y. Guo, T.B. Britton, A.J. Wilkinson. Acta Mater. 76 (2014) 1-12.

[20] T.B. Britton, A.J. Wilkinson. Acta Materialia 60 (2012) 5773-5782.

[21] J. Jiang, T.B. Britton, A.J. Wilkinson. Acta Mater. 61 (2013) 5895-5904.

[22] J. Jiang, T.B. Britton, A.J. Wilkinson. Acta Mater. 61 (2013) 7227-7239.

[23] J. Jiang, T.B. Britton, A.J. Wilkinson. Philosophical Magazine Letters 92 (2012) 580588. 
[24] T.B. Britton, H. Liang, F.P.E. Dunne, A.J. Wilkinson. Proc. R. Soc. A-Math. Phys. Eng. Sci. 466 (2010) 695-719.

[25] F.P.E. Dunne, R. Kiwanuka, A.J. Wilkinson. Proc. R. Soc. A-Math. Phys. Eng. Sci. 468 (2012) 2509-2531.

[26] T. Zhang, D.M. Collins, F.P.E. Dunne, B.A. Shollock. Acta Mater. 80 (2014) 25-38.

[27] M.E. Kartal, F.P.E. Dunne, A.J. Wilkinson. Acta Mater. 60 (2012) 5300-5310.

[28] S.J. Hessel, W. Voice, A.W. James, S.A. Blackham, C.J. Small, M.R. Winstone. Nickel alloy for turbine engine component. US Patent, 6132527, 2000.

[29] T.B. Britton, A.J. Wilkinson. Ultramicroscopy 114 (2012) 82-95.

[30] T.B. Britton, A.J. Wilkinson. Ultramicroscopy 111 (2011) 1395-1404.

[31] J. Nye. Acta metallurgica 1 (1953) 153-162.

[32] A.J. Wilkinson, D. Randman. Philosophical Magazine 90 (2010) 1159-1177.

[33] J. Jiang, T.B. Britton, A.J. Wilkinson. Ultramicroscopy 125 (2013) 1-9.

[34] L. Babout, Y. Brechet, E. Maire, R. Fougeres. Acta Mater. 52 (2004) 4517-4525.

[35] C. Tekog $\sim$ lu, T. Pardoen. Int. J. Plast. 26 (2010) 549-569.

[36] V.V.C. Wan, D.W. MacLachlan, F.P.E. Dunne. International Journal of Fatigue 68 (2014) 90-102.

[37] C.A. Sweeney, W. Vorster, S.B. Leen, E. Sakurada, P.E. McHugh, F.P.E. Dunne. J. Mech. Phys. Solids 61 (2013) 1224-1240.

[38] D. Dingley. Journal of Microscopy 213 (2004) 214-224.

[39] R.J. Arsenault, N. Shi. Materials Science and Engineering 81 (1986) 175-187.

[40] J. Jiang, T.B. Britton, A.J. Wilkinson. Int. J. Plast. 69 (2015) 102-117.

[41] A. Needleman. Journal of Applied Mechanics-Transactions of the Asme 54 (1987) $525-531$.

[42] X.P. Xu, A. Needleman. Modelling and Simulation in Materials Science and Engineering 1 (1993) 111-132. 
[43] V. Tvergaard. Mater. Sci. Eng. A-Struct. Mater. Prop. Microstruct. Process. 125

(1990) 203-213.

[44] C. Landron, O. Bouaziz, E. Maire, J. Adrien. Scr. Mater. 63 (2010) 973-976.

[45] M.P. Jackson, R.C. Reed. Materials Science and Engineering: A 259 (1999) 85-97.

(a)

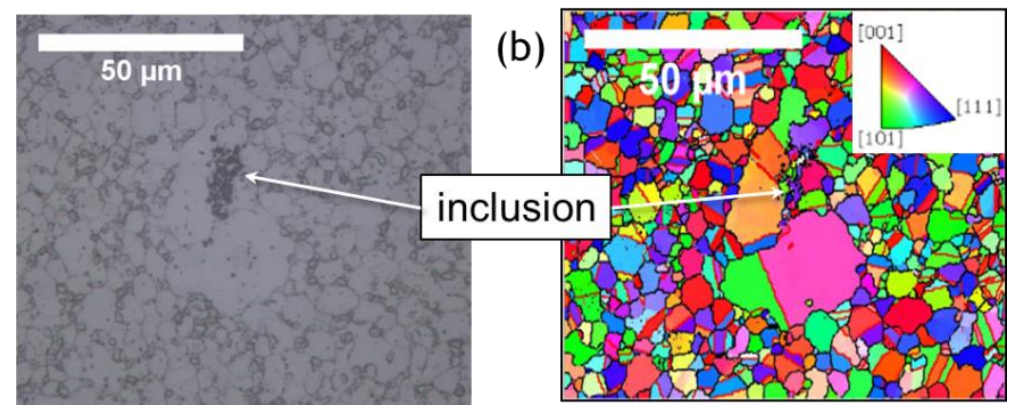

Figure 1 (a) EBSD orientation map with respect to the surface normal of the non-metallic inclusion agglomerate embedded in fine grain nickel superalloy. (b) An optical micrograph of the etched sample where primary $\gamma^{\prime}$ grains appear dark and can be seen at $\gamma$ boundaries.

(a)

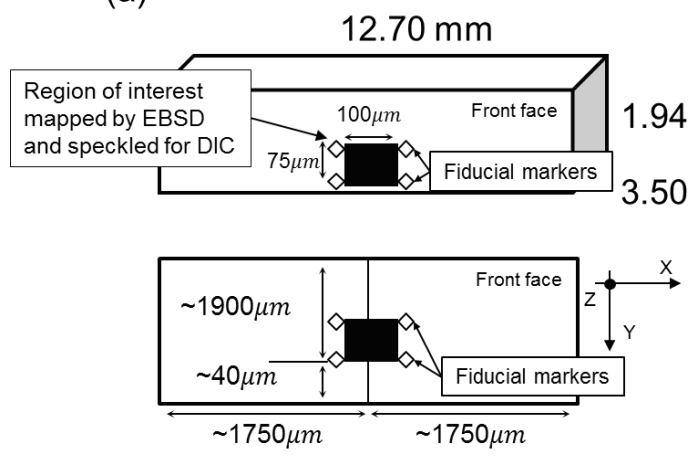

(b)

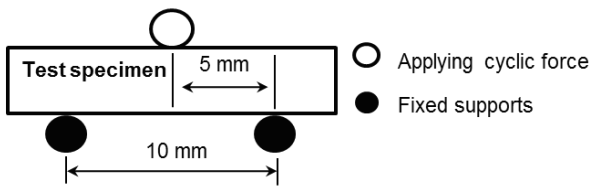

(c)

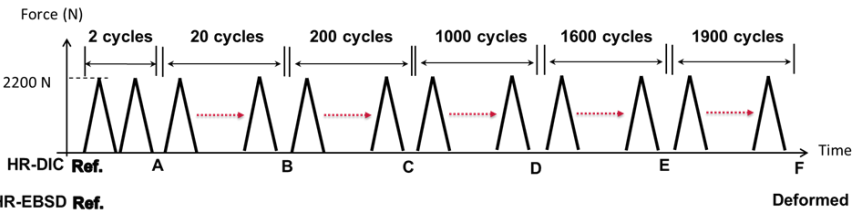

Figure 2 (a) Schematic representation of the specimen with its dimension (upper diagram). The location of inclusion agglomerate for EBSD and DIC on the front face is shown in the lower diagram. (b) A frontviewed schematic of the three-point bend test configuration. (c) The fatigue loading history and the intervals after which DIC and EBSD were conducted. 
(a)

(b)

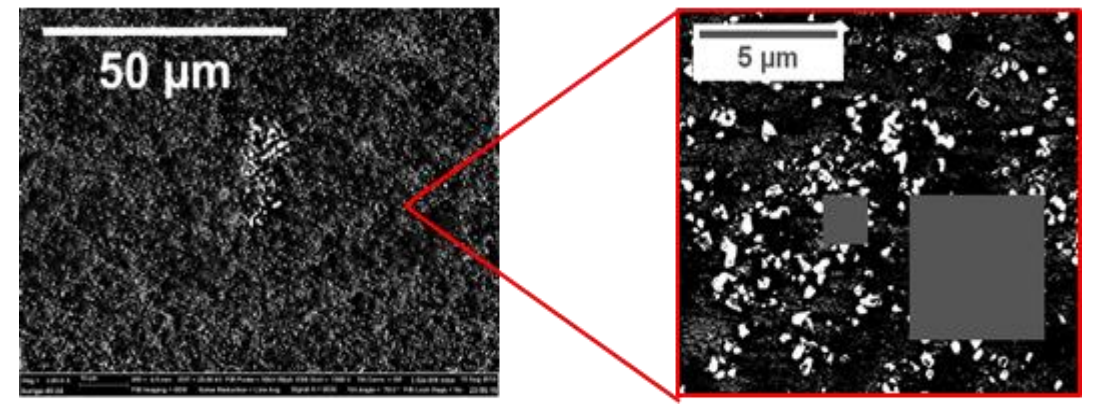

Figure 3 (a) The fine speckle pattern for DIC strain measurement with sub-grain resolution, (b) a magnified view showing the sizes of the sub sets used for the first two cycles (big square) and the remaining analysis (small square)
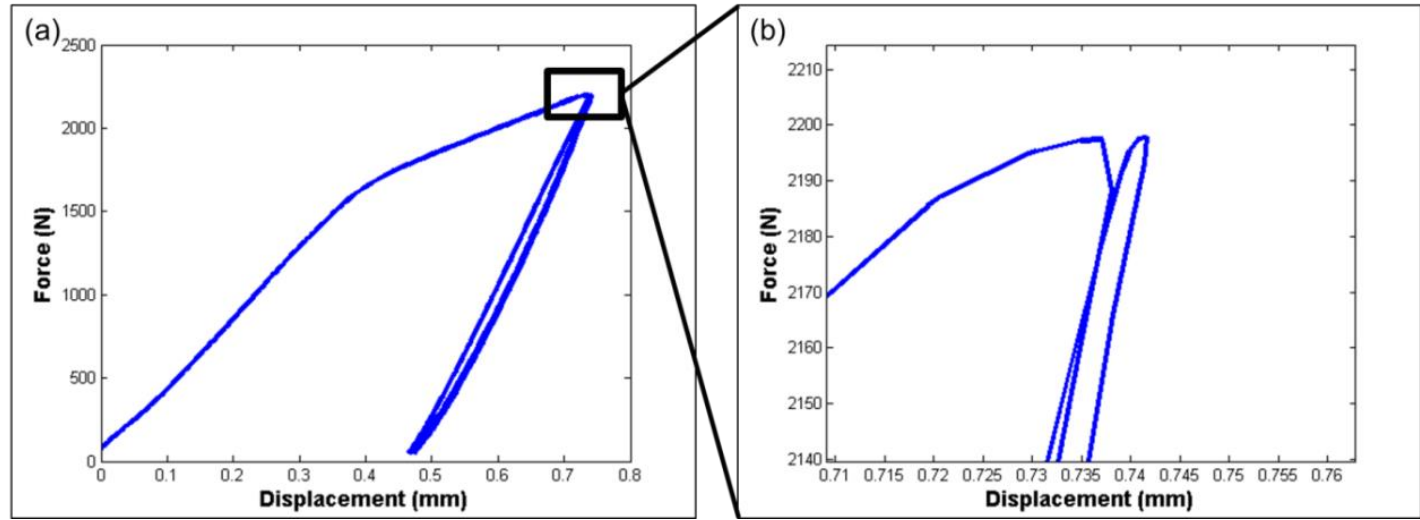

Figure 4 (a) Load displacement curve for the first two cycles of the force-controlled fatigue test with (b) a magnified plot showing an incremental increase in displacement. 


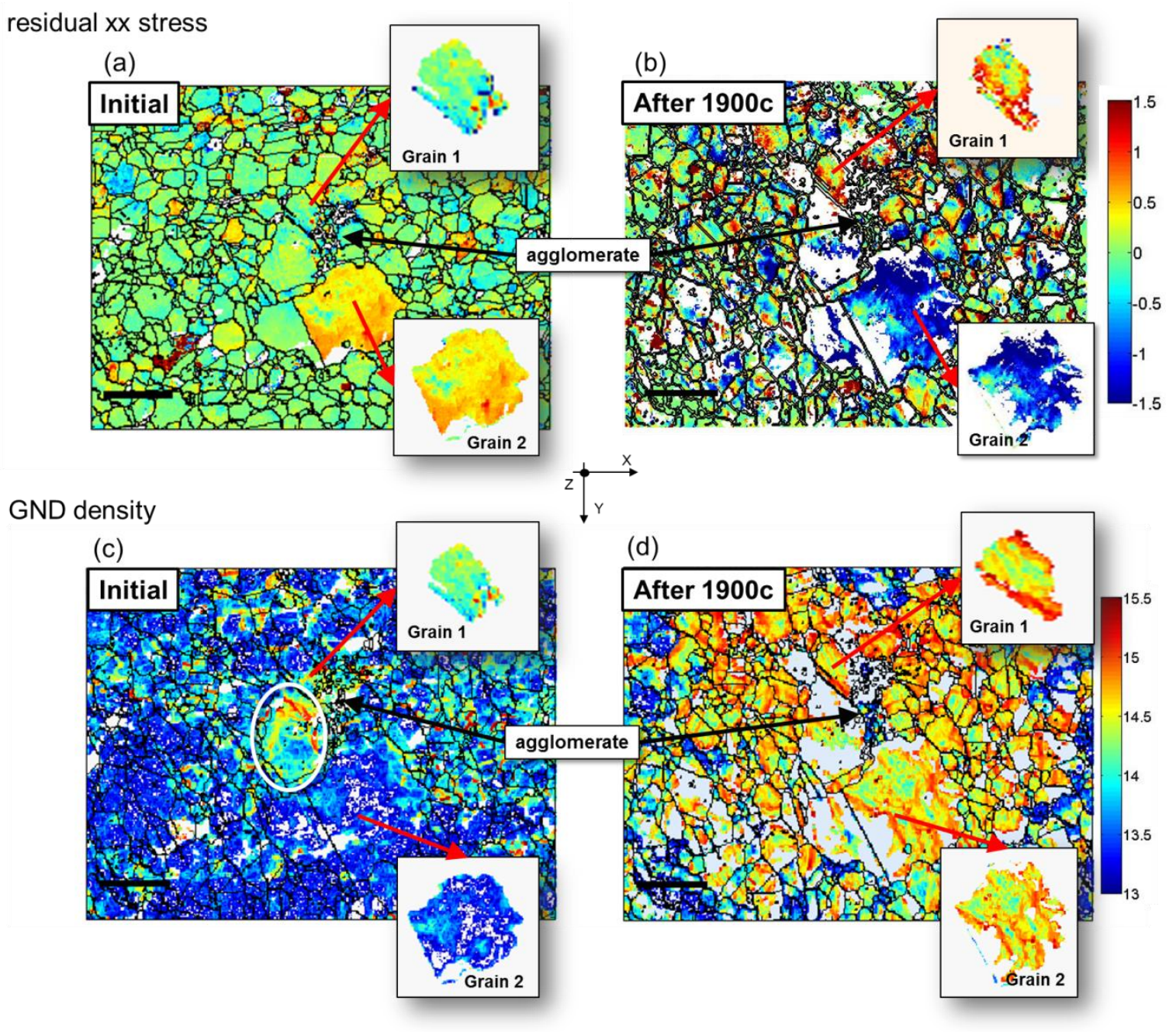

Figure 5 Field plots of HR-EBSD measured in-plane xx stress in GPa (a) prior to and (b) after the cyclic loading to 1900 cycles with grain boundaries marked using black lines, with these stress measurements with respect to references within each grain. HR-EBSD measured GND densities $\left(\log _{10}\right.$ (GND density in $\left.\mathrm{m}^{2}\right)$ )

(c) prior to and (d) after the fatigue. Two surrounding grains are selected for further quantitative analysis. Scale bar is $12.5 \mu \mathrm{m}$. Unresolved regions, due to poor correlation and high mean angular error are shown in white. The coordinate system is shown in the centre. 
(a)

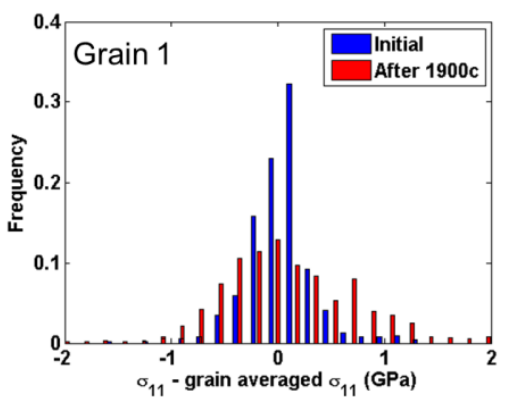

(b)

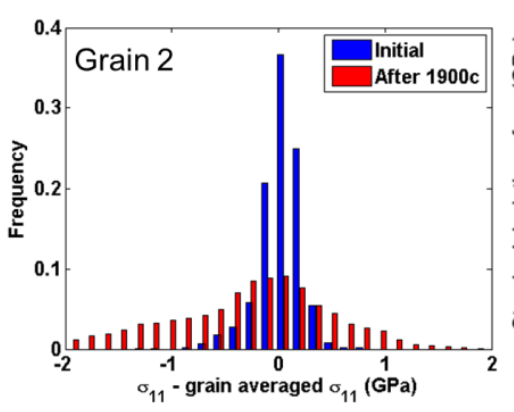

(c)

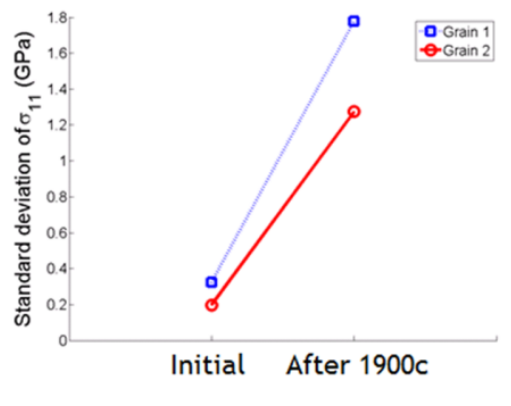

Figure 6 Histograms of grain averaged $\sigma_{11}$ for (a) grain 1 (b) grain 2 shown in Fig. 5, and (c) standard deviations prior to the cyclic straining and after 1900 cycles

(a)

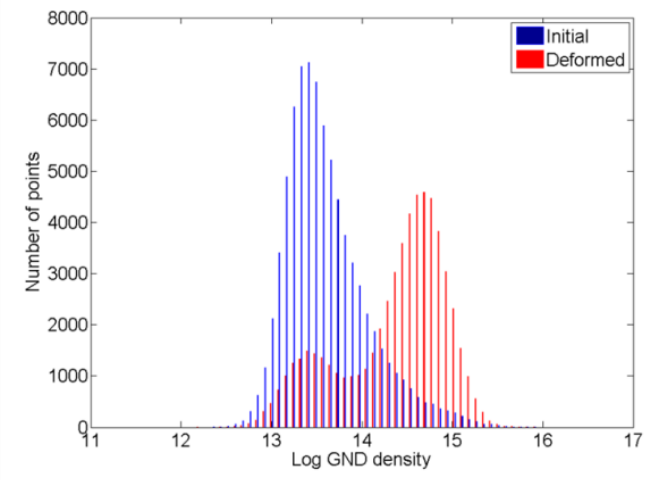

(b)

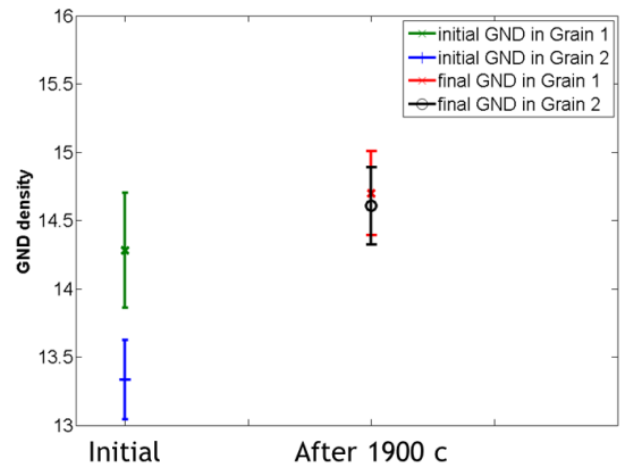

Figure 7 (a) Histograms of GND densities before the cyclic loading and after 1900 cycles. (b) Mean and standard deviation of the HR-EBSD measured GND density in Grain 1 and 2; prior to the cyclic straining and after 1900 cycles. GND density is in log (GND) $\mathrm{m}^{-2}$. Error bar is symmetric about mean value and is two standard deviation units in length 

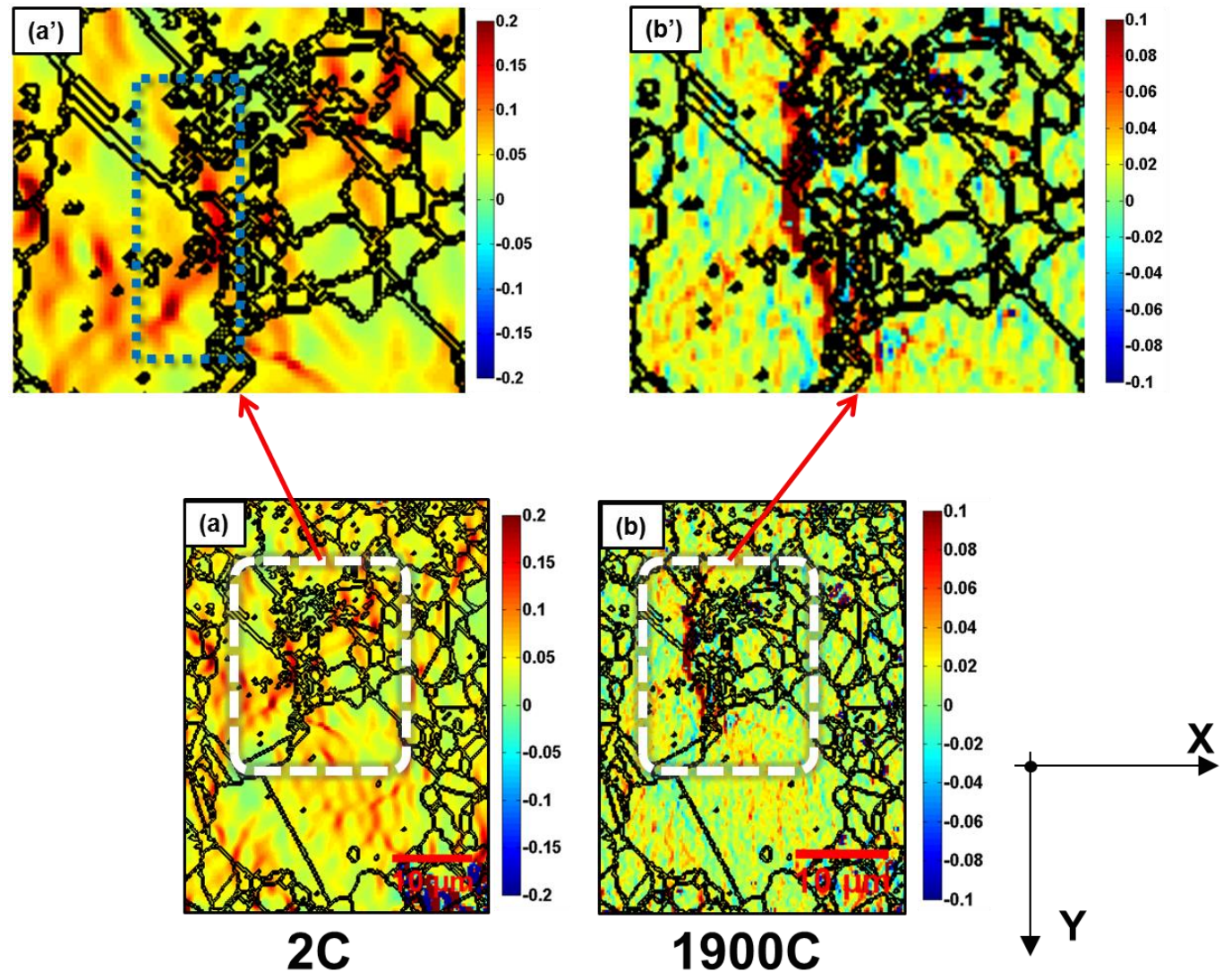

Figure 8 Contour plots of $x x$ incremental strains measured with HR-DIC (a) at 2, (b) 20 cycles with EBSD determined grain boundaries overlaid. A crack was first observed after 20 cycles via decohesion of agglomerate/nickel interface. Cracks coalesced and propagated into the surrounding nickel grains. The plotted strains are in the $x$ direction shown in Fig. 2.
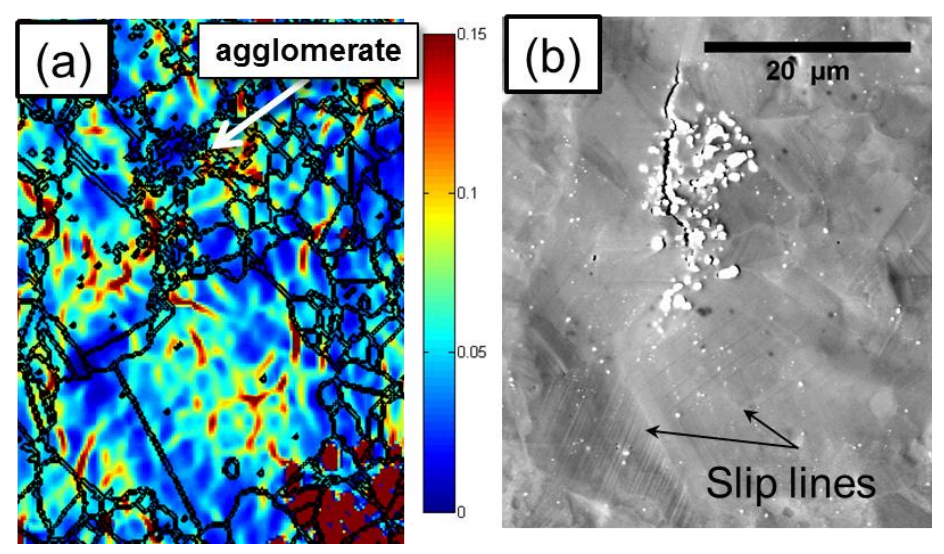

Figure 9 (a) Contour plot of effective plastic strain calculated between zero and two cycles. (b) BSE image taken after the fatigue loading. 


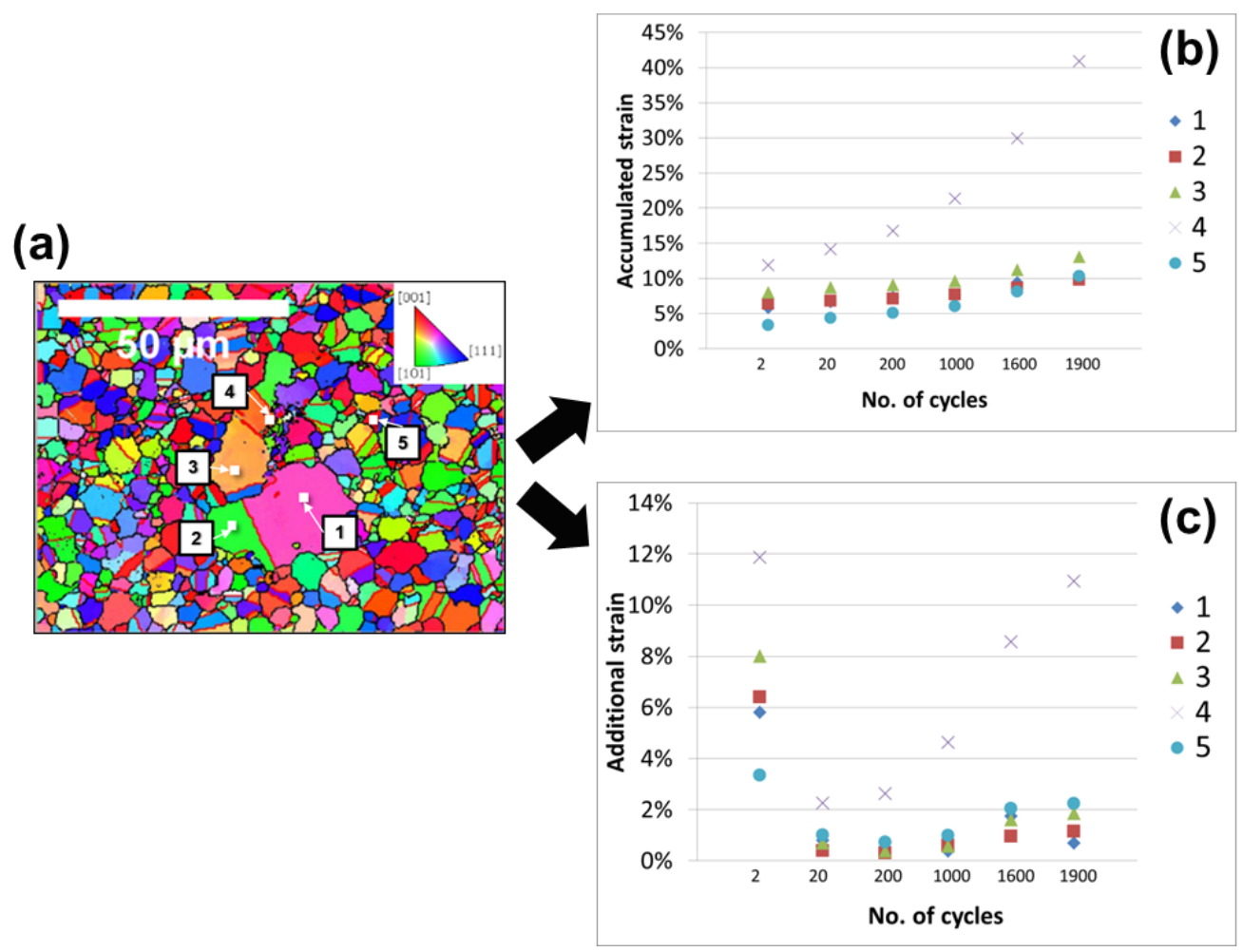

Figure 10 Strain evolution at the inclusion agglomerate, large surrounding grains, and fine grain as indicated by the points marked in the EBSD map (a). Strains in (b) correspond to the differences developed since the initial measurement. Incremental strains in (c) correspond to the differences developed since the previous measurement.

(a)

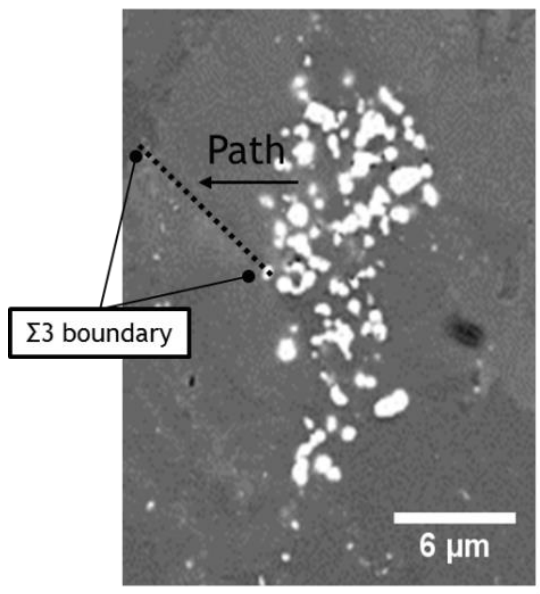

(b)

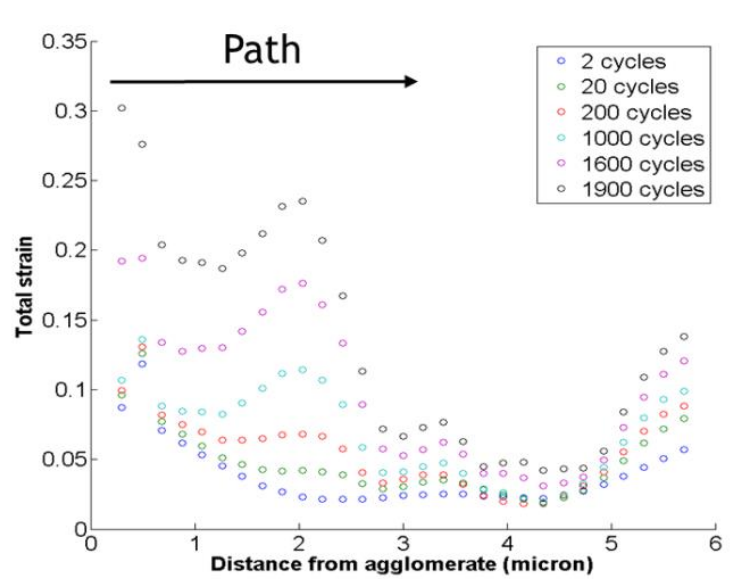

Figure 11 (a) A line scan (black arrow) from agglomerate proximity towards a $\Sigma 3$ grain boundary (dashed line). (b) The distribution of total strains along the line. The strains correspond to the differences since the initial measurement. 

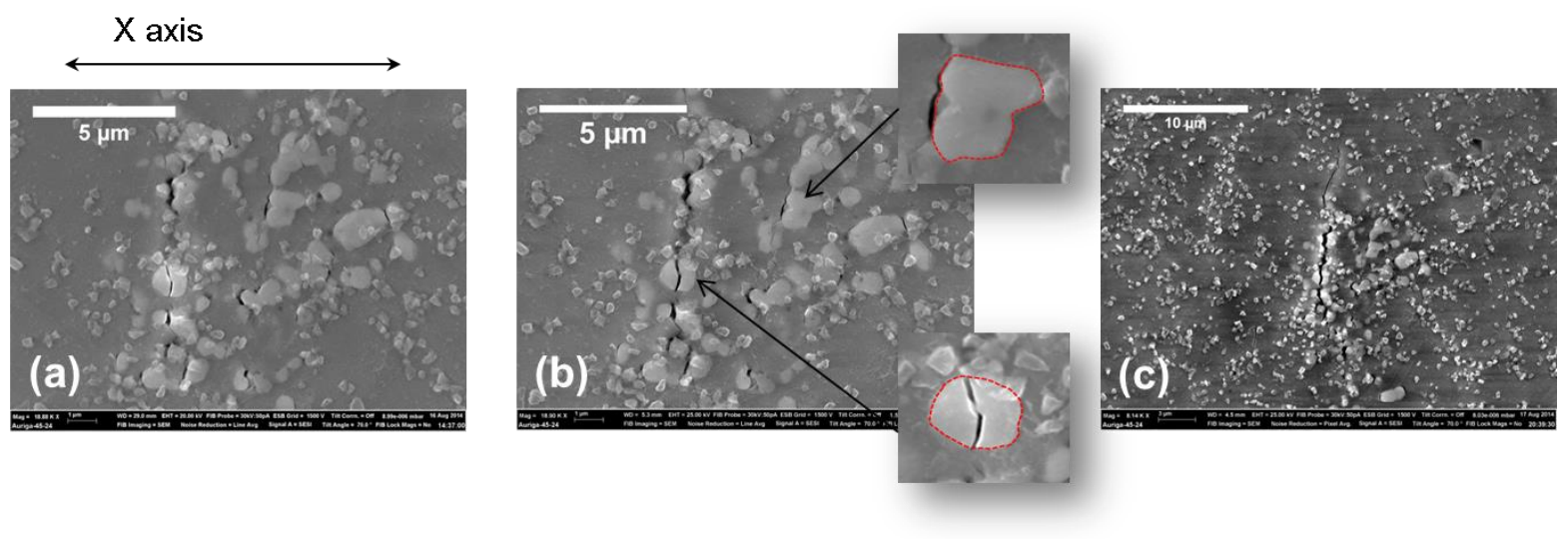

Figure 12 Microstructural evolution of fatigue cracks in the agglomerate. Cracks first observed after 20 cycles. (a) Cracking site after 1000 cycles. (b) The width of the cracks increases with insets showing interfacial decohesion and cracking of oxide particles. (c) Coalescence and propagation of the interconnected cracks into surrounding nickel grains. The fine particles are colloidal silica and the inclusion oxide particles are marked by the dashed red line.
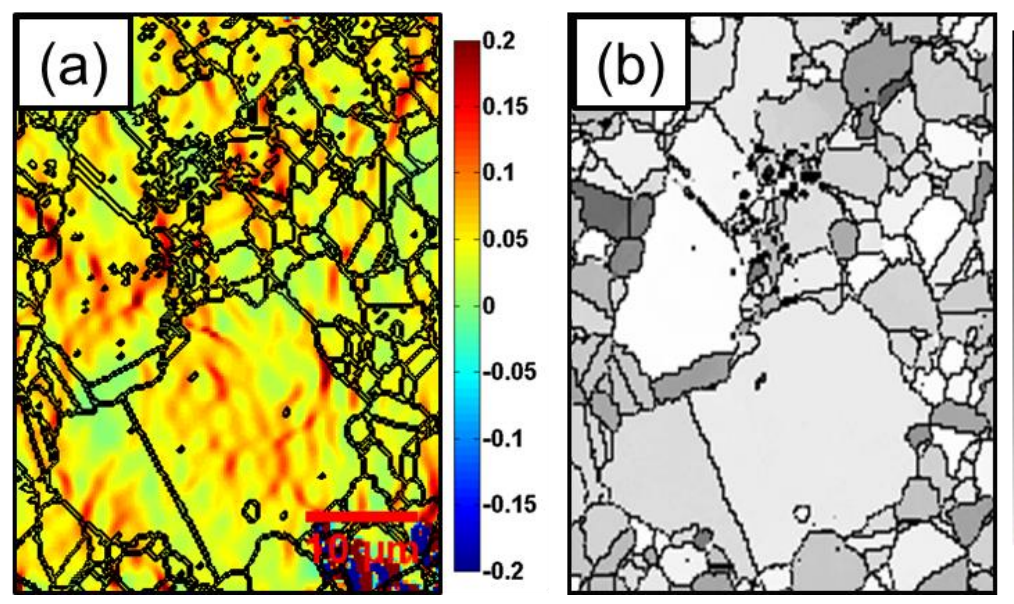

0.2

0.5

Figure 13 (a) Map of strain $x$ measured by DIC after 2 cycles. (b) Schmid factor map of the inclusion/matrix combination. Remote loading direction is horizontal, corresponding to the $x$ direction in Fig. 2. 12 fcc slip systems are considered. 

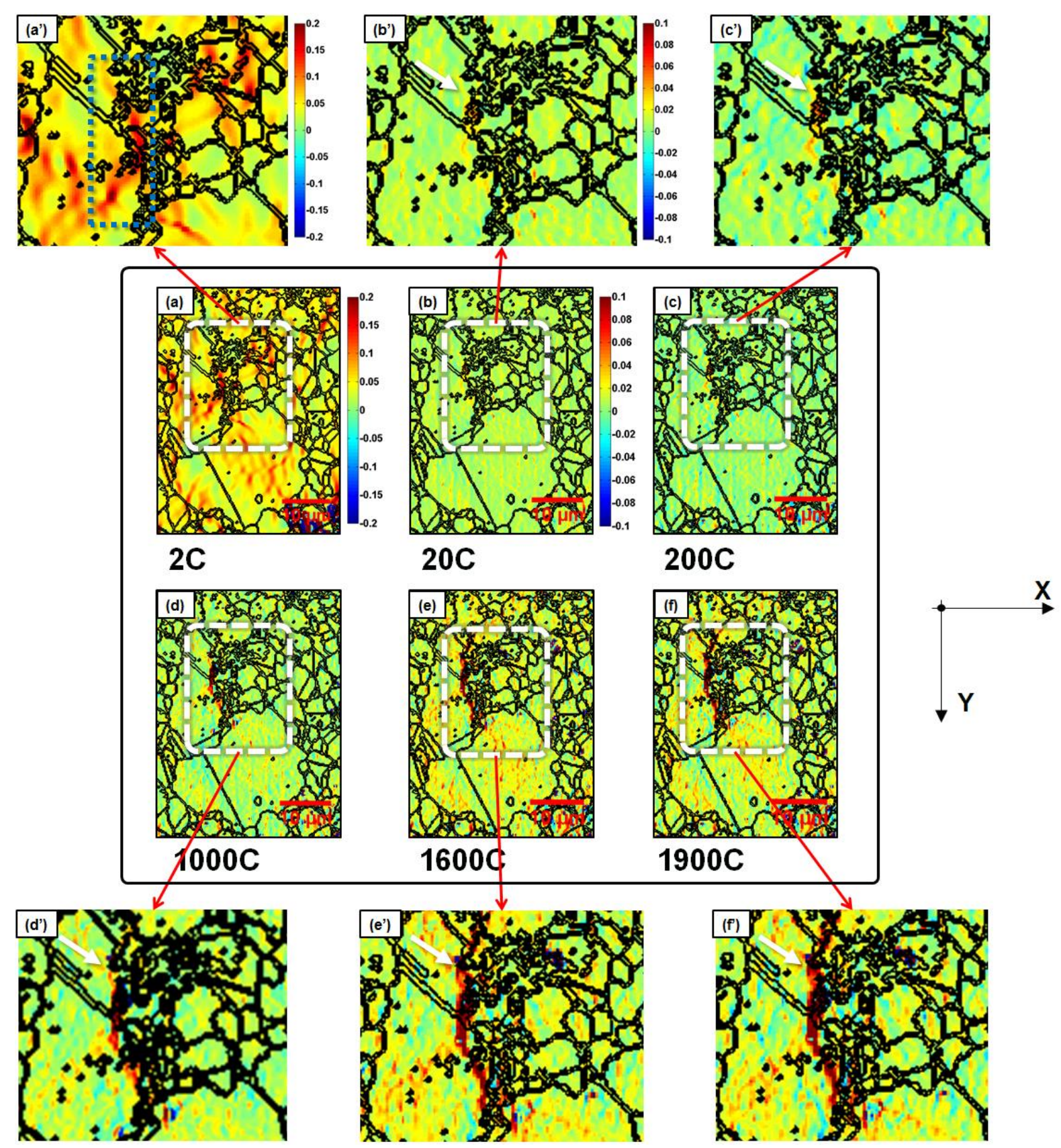

Figure 14 Contour plots of xx incremental strains measured with HR-DIC (a) at 2, (b) 20, (c) 200, (d) 1000,

(e) 1600, and (f) 1900 cycles with EBSD determined grain boundaries overlaid. A crack was first observed after 20 cycles via decohesion of agglomerate/nickel interface. Cracks coalesced and propagated into the surrounding nickel grains. The plotted strains are in the $\mathrm{x}$ direction shown in Fig. 2 . 